\title{
Cloning and expression of pluripotent factors around the time of gastrulation in the porcine conceptus
}

\author{
D.R. Eborn, D.L. Davis and D.M. Grieger \\ Animal Sciences and Industry, Kansas State University, Manhattan, KS, 66506 USA
}

The expression of the transcription factors Nanog, Sox-2 and Oct-4 is required for maintaining the inner cell mass and ensuing epiblast of the developing mouse embryo as well as pluripotency of embryonic stem cells in culture. Nanog and Oct-4 are down regulated about the time of gastrulation (Rosner et al. 1990, Chambers et al. 2003) whereas Sox-2 expression is observed in other tissues including the developing nervous system (Avilion et al. 2003). In embryonic stem cells, these factors suppress differentiation and promote self-renewal by forming an autoregulatory and feedforward network. The expression pattern of these markers in farm animal species is not well characterized and may differ from that of the mouse (Degrelle et al. 2005). Therefore, we have partially cloned the porcine Oct-4, Nanog and Sox-2 transcripts and characterized their expression in day-10,-12,-15, and -17 embryonic and extraembryonic tissues as well as endometrium, myometrium, placenta and fetal liver at day 40 of pregnancy (day 0 is the onset of estrus).

Embryos were flushed from sows 10, 12, 15, and 17 days post-insemination. Day-10 and -12 embryos were processed as whole conceptuses. Day-15 and -17 embryonic tissue (embryonic disk) was separated by closely trimming the adjacent extraembryonic tissue (proximal extraembryonic) with a scalpel using a stereo-microscope (5 to 50X). Additional extraembryonic tissue (distal extraembryonic) was collected after removal of the embryonic disks. Total RNA was isolated using RNeasy Mini or RNeasy Micro Kits (Qiagen; Valencia, CA) according to manufacture's instructions. Sequence for each transcription factor was obtained by full-length RNA ligase-mediated rapid amplification with either the RLM-Race (Ambion; Austin TX) or GeneRacer (Invitrogen; Carlsbad CA) kits according to manufacture's instructions. Total RNA was reverse transcribed and real-time PCR was peformed using TaqMan probe-based assays (Applied Biosystems; Foster City CA). Threshold values were normalized using 18 s ribosomal RNA as the endogenous control. Using the adjusted threshold values, tissue means were compared by the GLM procedure of SAS (SAS Institute Inc.; Cary NC) and pair-wise comparisons were made between tissues. For each gene, the tissue with the lowest adjusted threshold value was designated as the reference tissue. Relative expression differences were calculated by taking the difference in threshold values with the reference tissue and raising it by $2^{n}$.

The coding sequence for porcine Nanog (Genbank: DQ447201) including 452 base pairs of the Nanog promoter, and partial coding sequences of Oct-4 and Sox- 2 were obtained. The homeodomain and c-terminal tryptophan repeats are highly conserved in porcine Nanog compared to the mouse, human and bovine. In the promoter, the highly conserved Octamer and Sox binding sequences are also present.

Oct-4 and Sox-2 expression (see Table 1) was lowest in day-40 tissues except for fetal liver which was 20 and 71 fold, respectively, higher than endometrium. The pattern of Nanog RNA expression differed from Oct-4 and Sox-2. Day-40 tissues demonstrated the highest expression of Nanog, including endometrium ( 7 fold), fetal liver ( 27 fold), placenta (40 fold) and myometrium ( 72 fold) when compared to day-15 distal extraembryonic tissue. Expression of these transcription factors in fetal liver may indicate the presence of a stem cell population 
in the developing liver. This could coincide with hematopoiesis in the developing fetus. Expression in the endometrium, placenta and myometrium was unexpected. Expression of Nanog in adult mouse tissues has been reported (Hart et al. 2004) and the relatively high expression in these gravid tissues may be associated with rapid growth or other physiological responses in pregnancy.

Oct- 4 expression levels were similar for day-10, -12 and -15 conceptuses and disk but dropped 3 fold in day-17 disk. On the other hand, Sox-2 was up regulated 1000 fold in the day-15 disk and 2000 fold in the day- 17 disk when compared to the day- 12 conceptus. The up regulation of Sox-2 occurs when the initial neural structures are appearing in the disk.

Overall, the expression of Nanog, Oct-4 and Sox-2 in pig pregnancies reveals similarities to that in the mouse but expression in the early fetal period may indicate functions beyond early embryogenesis. Further study of the regulatory circuits at these stages is needed.

Table 1. Relative Expression of Nanog, Oct-4 and Sox-2 in the Conceptus and Gravid Uterus

\begin{tabular}{|c|c|c|c|c|}
\hline Tissue & n & Nanog & Oct-4 & Sox-2 \\
\hline D10 Conceptus & 3 & $7.8^{c}$ & $61.3^{\mathrm{d}}$ & $29.2^{\mathrm{de}}$ \\
\hline D12 Conceptus & 4 & $6.9^{c}$ & $52.8^{\mathrm{d}}$ & $21.9^{\mathrm{de}}$ \\
\hline D15 Embryonic Disk & 5 & $12.3^{\mathrm{ed}}$ & $65.7^{\mathrm{d}}$ & $1134.6^{t}$ \\
\hline D15 Proximal Extraembryonic & 5 & $3.9^{\mathrm{bx}}$ & $51.3^{\mathrm{d}}$ & $7.4^{\text {trod }}$ \\
\hline D15 Distal Extraembryonic & 5 & $1.0^{\mathrm{s}}$ & $8.5^{b}$ & $1.4^{\mathrm{ab}}$ \\
\hline D17 Embryonic Disk & 3 & $11.2^{\text {ed }}$ & $17.7^{\text {ted }}$ & $2022.6^{1}$ \\
\hline D17 Proximal Extraembryonic & 3 & $3.2^{\mathrm{abc} c}$ & $34.9^{\text {bed }}$ & $1.4^{\mathrm{ab}}$ \\
\hline D17 Distal Extraembryonic & 3 & $1.2^{\text {b }}$ & $8.9^{\mathrm{b}}$ & $3.9^{\mathrm{abc}}$ \\
\hline D40 Endometrium & 3 & $7.0^{r}$ & $1.3^{4}$ & $1.0^{2}$ \\
\hline D40 Myometrium & 3 & $72.6^{\mathrm{e}}$ & $1.0^{+}$ & $1.8^{\text {at }}$ \\
\hline D40 Liver & 3 & $27.4^{\text {the }}$ & $1.5^{\circ}$ & $71.5^{\mathrm{e}}$ \\
\hline D40 Placenta & 3 & $39.9^{\text {dte }}$ & $26.7^{\text {bed }}$ & $5.2^{\mathrm{dbad}}$ \\
\hline
\end{tabular}

abcetexpression values having different superscripts within column are different $(P<0.05)$

\section{References}

Avilion AA, Nicolis SK, Pevny LH, Perez, Vivian N \& Lovell-Badge R 2003 Multipotent cell lineages in early mouse development depend on SOX2 function. Cenes \& Development 17 126-140.

Chambers I, Colby D, Robertson M, Nichols J, Lee S, Tweedie S \& Smith A 2003 Functional expression cloning of Nanog, a pluripotency sustaining factor in embryonic stem cells. Cell 113 643-655.

Degrelle SA, Campion E, Cabau C, Piumi F, Reinaud P, Richard C, Renard JP \& Hue I 2005 Molecular evidence for a critical period in mural trophoblast development in bovine blastocysts. Developmental Biology 288 448-460.

Hart AH, Hartley L, Jbrahim M \& Robb L. 2004 Identification, cloning and expression analysis of the pluripotency promoting Nanog genes in mouse and human. Developmental Dynamics 230 187-198.

Rosner MH, Vigano MA, Ozato K, Timmons PM, Poirier F, Rigby PW \& Staudt LM 1990 A POU-domain transcription factor in early stem cells and germ cells of the mammalian embryo. Nature 345 686-692. 